\section{Prevalência de cárie dentária e condições socioeconômicas em jovens alistandos de Manaus, Amazonas, Brasil}

\author{
Prevalence of dental caries and \\ socioeconomic conditions among \\ young conscripts in Manaus, \\ Amazonas, Brazil
}

\author{
Alcione Regina Pegoraro Viana \\ Rosana Cristina Pereira Parente \\ Maria Rosa Borras \\ Maria Augusta Bessa Rebelo \\ Universidade Federal do Amazonas
}

Processo CAAE n. 0191.0.115.000-07

Correspondência: Maria Augusta Bessa Rebelo. Rua Rio Itannauá, 194/504, Nossa Sra das Graças,

Manaus, AM CEP 69053-040. E-mail: augusta@ufam.edu.br

\section{Resumo}

A cárie dentária tem origem multifatorial e a condição socioeconômica está inserida neste contexto. O objetivo deste estudo foi conhecer a prevalência de cárie, além de verificar sua associação com variáveis socioeconômicas em jovens alistandos de 17 a 19 anos de idade, sexo masculino, em Manaus, Amazonas, Brasil. Foi realizado um estudo transversal com uma amostra de conveniência dos conscritos das Forças Armadas Brasileiras. Os critérios de diagnóstico e questionário socioeconômico foram baseados no levantamento nacional SB Brasil e OMS. Uma única examinadora (kappa = $0,96)$ realizou o exame bucal em 578 alistandos. Foi utilizado o teste Shapiro-Wilk para verificação de normalidade dos dados; não sendo aceita a normalidade ( $p=0,0001)$, foi utilizado o teste não-paramétrico KruskallWalis para comparação das médias e assim verificar a diferença significativa entre CPOD e seus componentes com as variáveis avaliadas. A prevalência de cárie encontrada foi de $88,8 \%$ e o índice CPOD médio foi igual a 5,16 $\pm 0,17$. Diferenças estatisticamente significantes foram encontradas nas médias dos componentes Cariado, Perdido e Obturado do índice CPOD, sendo os piores indicadores verificados nos grupos de menor escolaridade, menor renda e procedentes de escola pública, demonstrando maior necessidade de medidas preventivas e assistenciais para estes grupos.

Palavras-chave: Cárie Dentária. Índice CPOD. Condições socioeconômicas. Adolescência. Epidemiologia. 


\section{Abstract}

Dental caries has multifactor origins and socioeconomic conditions are part of this context. The aim of this study was to determine the prevalence of caries and investigate its association with socioeconomic variables among young male conscripts aged 17 to 19 years in Manaus, Amazonas. This was a cross-sectional study using a convenience sample of conscripts of the Brazilian armed forces. Diagnostic criteria and the socioeconomic questionnaire were based on the $\mathrm{SB}$ Brazil national survey and WHO criteria. A single examiner (kappa $=0.96)$ performed oral examinations on 578 conscripts. The Shapiro-Wilk test was used to verify normal distribution, and for non-normally distributed data $(\mathrm{p}=0.0001)$, the nonparametric Kruskal-Wallis test was used to compare means. Any significant differences in the DMFT index and its components, relative to the variables evaluated, were investigated. The prevalence of caries was $88.8 \%$ and the mean DMFT index was $5.16 \pm 0.17$. Statistically significant differences were found in the means for decayed, missing and filled components of the DMFT index. Worse indicators were found in groups with lower schooling, lower income, and from public schools, thus demonstrating the need to improve prevention and care for these groups.

Keywords: Dental caries. DMFT index. Socioeconomic conditions. Adolescence. Epidemiology.

\section{Introdução}

Diversos estudos têm demonstrado uma expressiva redução na prevalência de cárie na maioria dos países desenvolvidos ${ }^{1-3}$. No Brasil, entre 1980 e 2003, a redução nos valores do índice CPOD mostra uma tendência consistente de queda ao longo do período. Tal redução é observada para o país como um todo e para as cinco regiões, evidenciando que todas contribuem para o declínio nacional do $\mathrm{CPOD}^{4-10}$.

O primeiro levantamento epidemiológico nacional, realizado em 1986, abrangeu a zona urbana de 16 capitais brasileiras e mostrou um índice de CPOD muito alto $(12,69)$ na faixa etária de 15 a 19 anos de idade $^{4}$. No levantamento de 2003, o processo de amostragem foi por conglomerado envolvendo 250 municípios distribuídos em portes populacionais, além da área rural, incluindo 50 cidades da região amazônica. O estudo apontou um CPOD médio de 6,14 nesta faixa etária. Assim, apesar das diferenças metodológicas entre os estudos, pode-se inferir que houve uma redução no índice de aproximadamente $50 \%$.

Esse declínio tem sido atribuído à exposição das pessoas ao flúor através de várias formas de aplicação, tais como fluoretação da água de abastecimento público (sobretudo no Sul e Sudeste) e introdução de dentifrícios (com maior impacto nas regiões Norte, Nordeste e Centro-Oeste), e ainda melhoria nas condições de saúde e qualidade de vida. Além disso, as mudanças no critério de diagnóstico de cárie têm sido apontadas como fatores responsáveis pelo declínio na prevalência de cárie no Brasil e no mundo ${ }^{10-12}$. No entanto, alguns municípios brasileiros, como Manaus, não foram beneficiados da mesma forma, pois o município não possui fluoretação na água de abastecimento ${ }^{13}$.

Por outro lado, apesar de a cárie dentária ser a doença bucal mais estudada em todo o mundo, a maioria das pesquisas concentrase em crianças na faixa etária de 12 anos de idade, sendo escassos os dados sobre a prevalência de cárie em adolescentes ${ }^{11,14}$. 
O serviço militar concentra a maior quantidade de adolescentes do sexo masculino num mesmo período, tornando-se uma oportunidade para estudos epidemiológicos. Portanto, tendo em vista essa escassez, o objetivo deste estudo foi delinear o perfil epidemiológico da cárie dentária em alistandos de 17 a 19 anos de idade no município de Manaus, mediante o conhecimento da prevalência segundo análise do índice CPOD, seus componentes e indicadores socioeconômicos.

\section{Metodologia}

O delineamento do estudo foi do tipo transversal para cárie dentária, cuja população de referência correspondeu a 14.715 alistandos, conforme informações do Sistema Eletrônico de Recrutamento Militar (SERMIL), no ano de 2007, do Exército Brasileiro. Para a realização do estudo foi selecionada uma amostra de conveniência, entre jovens de 17 a 19 anos de idade que se apresentaram para o processo de seleção das Forças Armadas em Manaus no ano de 2007.

Para obtenção do tamanho mínimo da amostra (402 indivíduos), optou-se por realizar um estudo piloto, com a finalidade de conhecer as medidas de variabilidade do objeto estudado (prevalência de cárie) ${ }^{15}$. As medidas de tendência central e dispersão da variável dependente cárie foram: média de 5,46 e desvio padrão de 4,15. Além disso, considerou-se um erro amostral de 0,4 (unidades de CPOD) e um nível de confiança de $95 \%$.

No período de 40 dias destinado aos exames médicos odontológicos dos alistandos pelas Forças Armadas Brasileiras, foram sorteados, diariamente, 20 jovens por meio de amostragem de conveniência. Todos os participantes deveriam ser alfabetizados, pois preencheriam questionários sobre história médica e condições socioeconômicas. Um total de 775 alistandos aceitou participar do estudo, porém a avaliação da história médica (critério de exclusão) não permitiu a participação de 190 voluntários
$(24,52 \%)$, e sete $(0,9 \%)$ foram excluídos por não preencherem o questionário socioeconômico. Assim sendo, a amostra final ficou constituída por 578 indivíduos.

O questionário sobre história médica foi utilizado contendo os critérios de exclusão da amostra. Eram excluídos os indivíduos com idade superior a 19 anos (20,30\%), os não residentes em Manaus (5,58\%), os que faziam uso de aparelho ortodôntico (2,53\%), e aqueles que utilizaram enxaguatório bucal $(63,96)$ ou antibiótico nos 7 dias anteriores ao exame $(4,06 \%)$.

Os dados socioeconômicos foram coletados através de um questionário, antes do exame clínico. As questões referentes à condição socioeconômica do alistando no instrumento foram baseadas no "Levantamento das Condições de Saúde Bucal no Brasil - Projeto SB 2003" (BRASIL, 2004). As questões abordavam o alistando sobre grau de instrução, tipo de escola e renda familiar. A renda familiar descrita pelo participante foi convertida para o número de salários mínimos - SM (1 SM = R \$380,00, em setembro de 2007).

Os alistandos foram examinados por uma única examinadora cirurgiã-dentista (A.R.P.V.), utilizando espelhos bucais e sondas periodontais (sonda CPI) esterilizados, seguindo os critérios de biossegurança. Ainda, uma lanterna de testa foi utilizada como auxílio na iluminação. Os indivíduos eram examinados sentados e a examinadora de pé, auxiliada por um anotador. Desta forma, as coletas dos dados clínicos foram feitas com modificações nas recomendações do SB Brasil ${ }^{6}$.

Para garantir a confiabilidade e reprodutibilidade dos dados foi realizado um prévio treinamento intra-examinador e teste de coeficiência Kappa em 20 jovens estudantes de odontologia da Universidade Federal do Amazonas, onde se observou uma concordância de $98 \%$ e estatística Kappa de 0,96, representando um nível satisfatório de concordância. Exercícios pré-teste do questionário foram realizados em 30 indivíduos da mesma idade, previamente ao estudo. 
Todos os jovens alistandos examinados assinaram um Termo de Consentimento Livre e Esclarecido. O presente estudo recebeu aprovação do Comitê de Ética em Pesquisas da Universidade Federal do Amazonas (Processo CAAE n. 0191.0.115.000-07).

Um banco de dados foi preparado e submetido à análise no programa Statistical Analysis Systems - SAS - versão 6.12 para microcomputador. Foi utilizado o teste não-paramétrico Kruskall-Walis. A adoção de teste não-paramétrico se justifica, pois o índice de cárie utilizado (CPOD) não apresentava distribuição normal. O nível de significância utilizado para todos os testes foi de $5 \%$.

\section{Resultados}

Participaram deste estudo 578 adolescentes do sexo masculino, alistandos das Forças Armadas Brasileiras, na faixa etária de 17 a 19 anos, sendo que a maioria com 18 anos de idade (65\%), enquanto $23 \%$ e $12 \%$ estavam com 17 e 19 anos, respectivamente..

O índice CPOD médio encontrado nesta população foi $5,16 \pm 0,17$. Dos 578 alistandos, 65 (11,2\%) não apresentaram lesão de cárie e $513(88,8 \%)$ apresentaram alguma experiência de cárie. Ainda, 50,9\% dos indivíduos não apresentaram nenhum dente perdido $(\mathrm{p}=0)$ e a média de dentes presentes na boca na população estudada foi de 27,9 dentes. Com relação à composição do índice CPOD, o componente obturado apresentou maior percentual $(40,10 \%)$, enquanto os componentes cariado e perdido representaram, respectivamente, $35,30 \% \mathrm{e}$ 24,60\% (Tabela 1).
No presente estudo, as variáveis socioeconômicas consideradas foram: escolaridade do indivíduo, renda familiar, se estudou ou não (Estudante), tipo de escola que frequentava/frequentou. Com o objetivo de verificar a existência de diferença significativa entre a média do CPOD e de seus componentes, com relação à escolaridade do alistando, sua renda familiar, se estudava e qual o tipo de escola, utilizou-se o teste de Kruskall-Wallis. Para a variável Estudante, em nenhum dos componentes e nem no próprio CPOD foi encontrada diferença significativa. Para as variáveis Renda familiar e Tipo de escola, o CPOD não mostrou diferença significativa, entretanto isto foi observado quando a análise foi feita por componentes $(p<0,05)$, conforme a Tabela 2.

Com respeito à escolaridade do alistando verificou-se que as maiores médias de CPOD foram observadas nos grupos com Ensino Fundamental incompleto e Ensino Superior que não diferiram significativamente entre si. Além disso, a média de CPOD daqueles com Ensino Fundamental incompleto diferiu significativamente das demais. Finalmente, todas as outras comparações não diferiram entre si. As médias dos componentes cariados $(2,40 \pm 0,20)$ e perdidos $(1,94 \pm 0,17)$ apresentaram-se maiores nos alistandos com Ensino Fundamental Incompleto, diferindo significativamente das demais, e a média do componente obturado foi menor nestes indivíduos com menor escolaridade $(1,50 \pm 0,16)$.

Verificou-se também que as maiores médias dos componentes cariado e perdido foram observadas nos grupos com menor

Tabela 1 - Índice CPOD e componentes do índice em alistandos de 17 a 19 anos, na cidade de Manaus.

Table 1 -DMFT index and its components in 17 to 19-year-old male conscripts, in the city of Manaus.

\begin{tabular}{lccc}
\hline Componentes & Média do Componente & Erro padrão & Percentual \\
\hline Cariado & 1,82 & $\pm 0,09$ & $35,30 \%$ \\
Perdido & 1,27 & $\pm 0,08$ & $24,60 \%$ \\
Obturado & 2,07 & $\pm 0,12$ & $40,10 \%$ \\
CPOD & 5,16 & $\pm 0,17$ & $100,00 \%$ \\
\hline
\end{tabular}


Tabela 2 - Índice CPOD e componentes segundo variáveis socioeconômicas em alistandos de 17 a 19 anos $(\mathrm{n}=578)$.

Table 2 - DMFT index and its components according to socioeconomic variables in 17 to 19-year-old male conscripts $(n=578)$.

\begin{tabular}{lcccccccc}
\hline Variáveis & \multicolumn{2}{c}{ Cariados } & \multicolumn{2}{c}{ Perdidos } & \multicolumn{2}{c}{ Obturados } & \multicolumn{2}{c}{ CPOD } \\
\hline Escolaridade & \multicolumn{2}{c}{$\mathrm{p}=0.0013$} & \multicolumn{2}{c}{$\mathrm{p}=0.0001$} & \multicolumn{2}{c}{$\mathrm{p}=0.01$} & \multicolumn{2}{c}{$\mathrm{p}=0.0291$} \\
Ensino fundamental imcompleto (170) & 2.40 & $(0.20)$ & 1.94 & $(0.17)$ & 1.50 & $(0.16)$ & 5.84 & $(0.32)$ \\
Ensino fundamental completo (66) & 1.62 & $(0.25)$ & 1.11 & $(0.19)$ & 1.89 & $(0.36)$ & 4.62 & $(0.50)$ \\
Ensino médio imcompleto (211) & 1.65 & $(0.15)$ & 0.98 & $(0.10)$ & 2.32 & $(0.23)$ & 4.95 & $(0.30)$ \\
Ensino médio completo (88) & 1.42 & $(0.19)$ & 0.94 & $(0.22)$ & 2.39 & $(0.35)$ & 4.75 & $(0.46)$ \\
Ensino superior (38) & 1.58 & $(0.29)$ & 0.87 & $(0.25)$ & 3.05 & $(0.45)$ & 5.50 & $(0.54)$ \\
Renda familiar & $\mathrm{p}=0.0149$ & $\mathrm{p}=0.0169$ & $\mathrm{p}=0.0006$ & $\mathrm{p}=0.84$ (NS) \\
Até 5 salários mínimos & 1.89 & $(0.11)$ & 1.33 & $(0.09)$ & 1.92 & $(0.14)$ & 5.14 & $(0.21)$ \\
Mais de 5 salários mínimos & 1.34 & $(0.16)$ & 0.99 & $(0.18)$ & 2.80 & $(0.28)$ & 5.13 & $(0.41)$ \\
Estudante & $\mathrm{p}=0.57(\mathrm{NS})$ & $\mathrm{p}=0.56(\mathrm{NS})$ & $\mathrm{p}=0.25(\mathrm{NS})$ & $\mathrm{p}=0.94$ (NS) \\
Estuda & 1.73 & $(0.20)$ & 122 & $(0.08)$ & 2.13 & $(0.13)$ & 5.12 & $(0.19)$ \\
Não estuda & 2.06 & $(0.27)$ & 1.48 & $(0.23)$ & 1.73 & $(0.25)$ & 2.27 & $(0.46)$ \\
Tipo de Escola & $\mathrm{p}=0.0088$ & $\mathrm{p}=0.0004$ & $\mathrm{p}=0.0061$ & $\mathrm{p}=0.60$ (NS) \\
Pública & 1.90 & $(0.10)$ & 1.33 & $(0.09)$ & 2.02 & $(0.13)$ & 5.25 & $(0.20)$ \\
Privada & 1.13 & $(0.22)$ & 0.49 & $(0.14)$ & 2.94 & $(0.38)$ & 4.57 & $(0.42)$ \\
\hline
\end{tabular}

* médias seguidas por letras distintas, nas colunas, diferem entre si no nível de significância de $5 \%$.

* mean levels followed by different letters in the columns differ, among themselves, at a $5 \%$ significance level.

NS - Não Significativo = non-significant

renda familiar (até 5 salários mínimos) e alistandos que estudaram em Escola Pública. Estes grupos também apresentaram as menores médias no componente obturado, diferindo significativamente das demais.

Os alistandos com Ensino Superior, maior renda familiar (superior à 5 salários mínimos) e que estudaram em escola privada apresentaram maior quantidade de dentes obturados, e também menor quantidade de dentes perdidos.

\section{Discussão}

Sabe-se que os dados sobre cárie dentária em adolescentes são escassos no Brasil $^{11,14,16}$ e acabam se restringindo, em grande parte, à faixa etária de 12 anos de idade. Assim, os alistandos das Forças Armadas podem ser uma alternativa nos estudos de saúde bucal que objetivam obter informações a respeito de adulto jovens do sexo masculino, assim como acontece em outros países desde o início do século $\mathrm{XX}^{2}$, além de outros estudos nacionais, pois se caracteriza como uma amostra representativa destes jovens da população geral do município.

Por outro lado, o CPOD médio em adolescentes encontrado em diferentes países é muito variável, desde 2,43 encontrado no estudo transversal de Hopcraft e Morgan $(2005)^{17}$, realizado com recrutas de 17 a 20 anos de idade na Austrália, até 13,9 na Ilha de Sirituba, PA, com adolescentes de 14 anos de idade ${ }^{18}$. Essas divergências podem ser atribuídas a diferenças na faixa etária, época realizada e local do estudo, e sobretudo pelo fato de que as médias mascaram diferenças socioculturais entre países ou dentro de um mesmo país, Estado ou município, fato que revela a importância de estudos locoregionais.

O presente estudo abordou jovens alistandos entre 17 e 19 anos, do sexo masculino e do município de Manaus, AM, e encontrou um CPOD médio de 5,16. Em 1986, o CPOD médio para a faixa etária de 15 a 19 anos na Região Norte foi de 11,89, e em 2003 foi de $6,17^{4,6}$. Desta forma, podemos sugerir que houve uma redução na prevalência da doença cárie em adolescentes de aproximadamente $50 \%$ na região Norte, no período 
de 1986 e 2003, bem como uma redução no índice quando abordamos apenas a população do atual estudo em Manaus. Entretanto, vale ressaltar a cautela necessária e a limitação ao se comparar estimativas obtidas por outros estudos, considerando que a falta de padronização e aferição das variáveis entre outros fatores, podem comprometer essa análise ${ }^{10}$.

O CPOD médio encontrado nos alistandos mostra-se um pouco superior ao observado em escolares da cidade de Manaus, na faixa etária de 15 a 19 anos $(4,65)^{19}$. Entretanto, está próximo ao encontrado por Gomes et al. (2004) ${ }^{20}$ em jovens de 18 anos do município de Paulínia, SP $(5,3)$, e abaixo do verificado em adolescentes, na faixa etária de 15 a 19 anos, no município do interior do Amazonas, Rio Preto da Eva $(6,23)^{21}$, e em jovens de 17 a 19 anos do Estado de São Paulo $(6,44)^{11}$.

Ao se comparar o índice CPOD da população do nosso estudo $(5,16)$ com o obtido para a população de 12 anos do município $(2,81)^{22}$, verifica-se um incremento anual de 0,47 dentes atacados pela cárie, sendo este superior ao encontrado em recrutas de Santa Catarina $(0,39)$. Isso demonstra, além de uma tendência de crescimento na prevalência em função da idade, assim como no estudo nacional SB Brasil, também um possível impacto da não fluoretação da água de abastecimento ${ }^{6}$.

$\mathrm{O}$ valor do componente perdido $(\mathrm{P}$ $=1,27$ ) foi semelhante ao encontrado no último levantamento nacional, $\mathrm{P}=1,34$ (6), porém muito superior ao encontrado em recrutas da região Sul, Sudeste, Distrito Federal e Suécia, com menos de 0,3, $0,42,0,61$ e 0,1 , respectivamente ${ }^{14,11,23,24}$. Os maiores índices de dentes cariados e perdidos concentraram-se nos grupos de menor escolaridade e renda, revelando que as condições de saúde bucal expressam as desigualdades sociais.

O CPOD médio encontrado nos alistandos entre 17 e 19 anos de idade foi próximo ao encontrado em recrutas da Austrália $(\mathrm{CPOD}=5,15)$, na mesma faixa etária não exposta à água fluoretada, signi- ficativamente maior do que os indivíduos expostos ao flúor $(\mathrm{CPOD}=3,8)^{18}$. A água de abastecimento da cidade Manaus não é fluoretada e chega a $74,5 \%$ dos moradores do município ${ }^{25}$ e o restante consome água de poços artesianos e outras fontes. Segundo Santos et al. (2006) ${ }^{13}$, a concentração de flúor encontrada em poços semi-artesianos de diversos pontos foi desprezível, o que indica que a população não faz uso rotineiro de água fluoretada. Assim, um programa de fluoretação da água de abastecimento poderia favorecer uma melhora na condição de saúde bucal em toda população do município, principalmente aos mais expostos à iniquidades.

O valor percentual de alistandos com experiência de cárie $(88,8 \%)$ está um pouco aquém das encontradas em Paulínia, SP $(90,4 \%)^{11}$, em $2004(93,9 \%)^{20}$, e se assemelha à prevalência encontrada no estudo de Rebelo et al. $(2009)^{19}(87,4 \%)$ realizado com escolares, de ambos os sexos, na faixa etária de 15 a 19 anos, na cidade de Manaus. Além disso, o valor encontrado mostrase um pouco mais alto que os estudos com recrutas em Florianópolis, em $2002^{14}$ (81\%), Maringá, em $2005^{16}$ e em jovens de Estocolmo, Suécia ${ }^{26}$ (81\%), sendo ainda considerados elevados. No entanto, todos foram maiores que a prevalência de $67,2 \%$ registrada na Austrália, em recrutas de 17 a 20 anos de idade ${ }^{27}$.

A presença de $85 \%$ da população de 18 anos com todos os dentes naturais presentes foi a meta estabelecida pela OMS para o ano 2000 , aumentando para $100 \%$ de ausência de dentes perdidos para o ano de $2020^{28}$. O número médio de dentes presentes na boca dos alistandos examinados foi de 27,9 dentes. A porcentagem da população estudada com 28 dentes ou mais $(\mathrm{p}=0)$ foi igual a $50,9 \%$, um pouco mais elevada que o resultado dos dois levantamentos nacionais para a região Norte, $32 \%$ e $39,13 \%$, respectivamente ${ }^{4,6}$, porém muito aquém dos estudos com recrutas na região Sul, Florianópolis e Maringá ${ }^{14,16}$, e Distrito Federal ${ }^{23}$. O estudo realizado com estudantes em Paulínia, no Sudeste, foi um dos únicos que 
mostrou atingir a meta da OMS para o ano 2000 , mesmo assim com atraso de quatro $\operatorname{anos}(85,7 \%)^{20}$.

Manaus é a quarta cidade do país em geração de riqueza nacional, porém apresenta baixos indicadores sociais, demonstrando sua desigualdade social $^{29}$. Apesar de o estudo não apresentar diferenças estatisticamente significativas entre as variáveis socioeconômicas avaliadas e o índice CPOD, pode-se verificar que as maiores médias dos componentes Cariado e Perdido do índice CPOD concentraram-se nos indivíduos com menor grau de escolaridade e renda familiar, estando de acordo com alguns estudos $^{14,16,23,26,30-33}$. Além disso, apesar da semelhança no índice CPOD dos indivíduos com ensino Fundamental Incompleto $(5,84)$ e Ensino Superior $(5,5)$, observou-se que o componente obturado dos indivíduos com maior grau de escolaridade mostra-se muito mais elevado, sendo responsável por $55,45 \%$ do índice; enquanto que indivíduos com menor grau de escolaridade, ensino Fundamental Incompleto, tiveram 74,32\% dos dentes atacados por cárie, estando estes perdidos ou cariados. Assim, pode-se suge- rir que indivíduos com maior escolaridade têm mais facilidade de acesso ao tratamento odontológico.

Vale ressaltar que a não observância na diferença do índice CPOD e grau de escolaridade, pode estar relacionada ao fato de a amostra ter sido calculada para prevalência de cárie e não para escolaridade.

\section{Conclusão}

O estudo demonstrou que $88 \%$ dos alistandos de 17 a 19 anos na cidade de Manaus apresentavam experiência de cárie. $\mathrm{O}$ valor do índice CPOD encontrado foi igual a 5,16. A análise das variáveis "grau de escolaridade" e "renda familiar" demonstraram que os indivíduos com ensino fundamental incompleto e renda familiar $<5$ salários mínimos apresentaram as piores condições em relação à cárie dentária. Este fato alerta que a cárie dentária em adolescentes ainda constitui um problema de saúde pública e, também, que os agravos e a desigualdade na distribuição se intensificam em uma determinada parcela da população, a qual requer maior atenção em saúde bucal.

\section{Referências}

1. Petersson HG; Bratthall D. The caries decline: a review of reviews. Eur J Oral Sci 1996; 104: 436-43.

2. Ankkuriniemi O, Ainamo, J. Dental health and dental treatment needs among recruits of Finnish Defence Forces, 1919-91. Acta Odotol Scand 1997; 55: 192-7.

3. Antoft P, Rambusch E, Antof B, Christensen, HW. Caries experience, dental health behaviour and social status - Three comparative surveys among Danish military recruits in 1972, 1982 and 1993. Community Dent Oral Epidemiol 1999; 16: 80-4.

4. Brasil, Ministério da Saúde - Divisão de Saúde Bucal. Levantamento Epidemiológico em Saúde Bucal: Brasil zona urbana. 1986. Série C: estudos e projetos; 1988.

5. Brasil. Ministério da Saúde. Secretaria de Assistência à Saúde. Departamento de Assistência e Promoção da Saúde. Coordenação de Saúde Bucal. Levantamento epidemiológico em saúde bucal: $1^{a}$ etapa: cárie dental: projeto. Brasília; 1996.
6. Brasil. Ministério da Saúde. Secretaria de Atenção à Saúde. Departamento de Atenção Básica. Projeto SB Brasil 2003: condições de saúde bucal da população brasileira 2002-2003. Resultados Principais. Brasília; 2004.

7. Baldani MH, Narvai PC, Antunes, JLF. Cárie dentária e condições sócio-econômicas no Estado do Paraná, Brasil, 1996. Cad Saúde Pública 2002; 18: 755-63.

8. Cangussu MCT, Castellanos RA, Pinheiro MF, Albuquerque SR, Pinho C. Cárie dentária em escolares de 12 e 15 anos de escolas públicas e privadas de Salvador, Bahia, Brasil, em 2001. Pesqui Odontol Bras 2002; 16: 379-84.

9. Bastos JLD, Nomura LH, Peres MAA. Tendência de cárie dentária em escolares de 12 e 13 anos de idade de uma mesma escola no período de 1971 a 2002, em Florianópolis, Santa Catarina, Brasil. Cad Saúde Pública 2004; 20: 117-22. 
10. Narvai PC, Frazão P, Roncalli AG, Antunes, JLF. Cárie dentária no Brasil: declínio, polarização, iniqüidade e exclusão social. Rev Panam Salud Publica 2006; 19: 38593.

11. Gushi LL, Soares MC, Forni TIB, Vieira V, Wada RS, Sousa MLR. Cárie dentária em adolescentes de 15-19 anos de idade no Estado de São Paulo, Brasil, 2002. Cad Saúde Pública 2005; 21: 1383-91.

12. Antunes JLF, Peres MA, Mello TRC, Waldman EA. Multilevel assessment of determinants of dental caries experience in Brazil. Community Dent Oral Epidemiol 2006; 34: 146-52.

13. Santos FC, Pinheiro CS, Pereira JV, Parente RCP, Rebelo MAB. Avaliação da concentração de flúor em poços artesianos do município de Manaus - AM. Braz Oral Res 2006 Supplement; 20: 50.

14. Gonçalves ER, Peres MA, Marcenes W. Cárie dentária e condições sócio-econômicas: um estudo transversal com jovens de 18 anos de Florianópolis, Santa Catarina, Brasil. Cad Saúde Pública 2002; 18: 699-706.

15. Antunes JLF, Peres MA. Fundamentos de Odontologia: Epidemiologia da Saúde Bucal. Rio de Janeiro: Guanabara-Koogan; 2006.

16. Amaral MA, Nakama L, Conrado CA, Matsuo T. Cárie dentária em homens jovens: prevalência, severidade e fatores associados. Braz Oral Res 2005; 19: 249-55.

17. Hopcraft, M; Morgan, M.V. Dental caries experience in a young adult military population. Aust Dent J 2003; 48: $125-9$.

18. Normando ADC, Araújo IC. Prevalência de cárie dental em uma população de escolares da região amazônica. Rev Saúde Pública 1990; 24: 294-9.

19. Rebelo MAB, Lopes MC, Vieira JMR, Parente RCP. Dental caries and gingivitis among 15 to 19 year-old-students in Manaus-Am, Brazil. Braz Oral Res 2009; 23: 248-54.

20. Gomes PR, Costa SC, Cypryano S, Souza MLR. Paulínia, São Paulo, Brasil: situação da cárie dentária com relação às metas OMS 2000 e 2010. Cad Saúde Pública 2004; 20: 8555-70.

21. Lima RTS. Estudo da prevalência da cárie dental em Rio Preto da Eva, Amazonas - Brasil [dissertação de mestrado]. Manaus: Universidade Federal do Amazonas e Fundação Oswaldo Cruz - FIOCRUZ; 2007
22. Moura RNV. Prevalência de cárie e fluorose dentária em escolares do município de Manaus [dissertação de mestrado]. Manaus: Universidade Federal do Amazonas; 2004

23. Pádua, E.M.C.; Pinheiro, J.A. Avaliação do perfil de saúde bucal dos militares recrutas no batalhão da polícia do Exército de Brasília (2003). Brasília, DF: ABO; 2004.

24. Julihn A, Agholme MB, Grindefjord M, Modéer T. Risk factors and risk indicators with high caries experience in Swedish 19-year-olds. Acta Odontol Scand 2006; 64: 267-73.

25. IBGE - Instituto Brasileiro de Geografia e Estatística. Censo, 2000. Disponível em www.ibge.gov.br/ [Acessado em 20 de janeiro de 2007]

26. Hjern A, Grindefjord M, Rosén M. Social Inequality in oral health and use of dental care in Sweden. Community Dent Oral Epidemiol 2001: 29: 167-74.

27. Hopcraft M. Dental caries experience in Australian Army recruits 2002-2003. Aust Dent J 2005; 50: 16-20.

28. Hobdell M, Petersen PE, Clarkson J, Johnson N. Global goals for oral health 2020. Int Dent J 2003; 53: 285-88.

29. NUCPAM - Núcleo de Cultura Política do Amazonas, 2009. Disponível em www.nucpam.com/2009/07 [Acessado em 13de julho de 2009]

30. Gushi LL, Soares MC, Forni TIB, Vieira V, Wada RS, Sousa MLR. Relationship between dental caries and socioeconomic factors in adolescents. J Appl Oral Sci 2005; 13: 305-11.

31. Senna A, Campus G, Cagliani, Strohmenger L. Socioeconomic influence on caries experience and CPITN values among a group of Italian call-up soldiers and cadets. Oral Health Prev Dent 2005; 3: 39-46.

32. Kelbauskas E, Keubauskiené S, Paipaliené P. Smoking and others factors influencing the oral health of Lithuanian Army recruits. Military Medicine 2005; 170: 791-6.

33. Boing AF, Peres MA, Kavaleski DF, Zange SE, Antunes JLF. Estratificação sócio-econômica em estudos epidemiológicos da cárie dentária e doenças periodontais: características da produção na década de 90. Cad Saúde Pública 2005; 21: 673-78.

Recebido em: 01/06/09 Versão final reapresentada em: 09/10/09 Aprovado em: 23/10/09 